\title{
Использование MS Excel для обработки неразделенных пиков на хроматограммах
}

\author{
(C) 2020 Дейнека В.И. ${ }^{1}$, Туртыгин А.В. ${ }^{2}$, Нгуен Ван Ань ${ }^{3}$, \\ Олейниц Е.Ю. ${ }^{1}$, Дейнека Л.А. ${ }^{1}$ \\ ${ }^{I}$ ФГАОУ ВО «Белгородский государственный национальный исследовательский университет», \\ Белгород \\ ${ }^{2}$ Федеральное автономное учреждение «Национальный Институт Аккредитации», Москва \\ ${ }^{3}$ Ханойский педагогический университет 2, Ханой, Вьетнам
}

Поступила в редакцию 27.02.2020 г.

DOI: $10.17308 /$ sorpchrom.2020.20/2779

В работе на примере триацилглицеринов льняного масла и антоцианов плодов винограда сорта Молдова предложен способ компьютерного моделирования не полностью разделенных пиков с использованием программы MS Excel. Благодаря предложенному способу для неразделенных пиков возможен расчет таких параметров как число теоретических тарелок, разрешение пиков, количественное определение не полностью разделяющихся веществ. Полученные результаты сопоставляются с результатами, получаемыми стандартными для программного обеспечения хроматографов способами. Показано, что при существенном различии «полуширин» неразделенных пиков корректное соотношение между их содержанием может быть рассчитано только после их разделения гауссовыми функциями. Установлено, что дополнительное уширение возможно в том случае, если пик объединяет два изомера (например, изомеров положения триацилглицеринов) с небольшим различием удерживания между ними. Эмпирически установлено, что небольшое отклонение хроматографических пиков исследованных объектов от Гауссового распределения может быть компенсировано добавлением небольшого пика с большим временем удерживания, параметры которого могут быть подобраны при компьютерном моделировании пика в программе MS Excel.

Ключевые слова: ВЭЖХ, компьютерное моделирование пиков, MS Excel, гауссиан, неразделенные пики, количественный анализ.

\section{Введение}

В настоящее время для записи, обработки и хранения хроматограмм используются специализированные компьютерные программы, позволяющие производить не только запись, но и хранение, и обработку хроматограмм. Однако ряд операций при этом чаще всего остается недоступным. Так, например, при наложении двух или более пиков с разрешением $\left(\mathrm{R}_{\mathrm{s}}\right)$ менее 1 программное обеспечение Agilent ChemStation обычно дает сбой из-за ошибочного (по понятным причинам) детектирования начала или окончания пиков, рис. 1. Разумеется, в таких случаях необходима ручная переразметка пиков, включаемая в ПО Agilent ChemStation.

В трех широко используемых в хроматографической практике программных продуктах, доступных в нашей лаборатории (ChemStation 32 Agilent, LC и Мультихром 1.5), нет возможности автоматического разделения пиков моделированными гауссианами. Но для компьютерной обработки не полностью разделенных (называемых «критическими парами» при ВЭЖХ триацилглицеринов [1]) пиков можно вос- 
пользоваться программой MagicPlot, или бесплатной не полнофункциональной Student-версией программы [2]. Разделение таких пиков моделированными гауссианами позволит определить основные параметры пиков: время удерживания, так называемую «полуширину» и число теоретических тарелок, рассчитать разрешение пиков (степень разделения), $\mathrm{R}_{\text {s. }}$

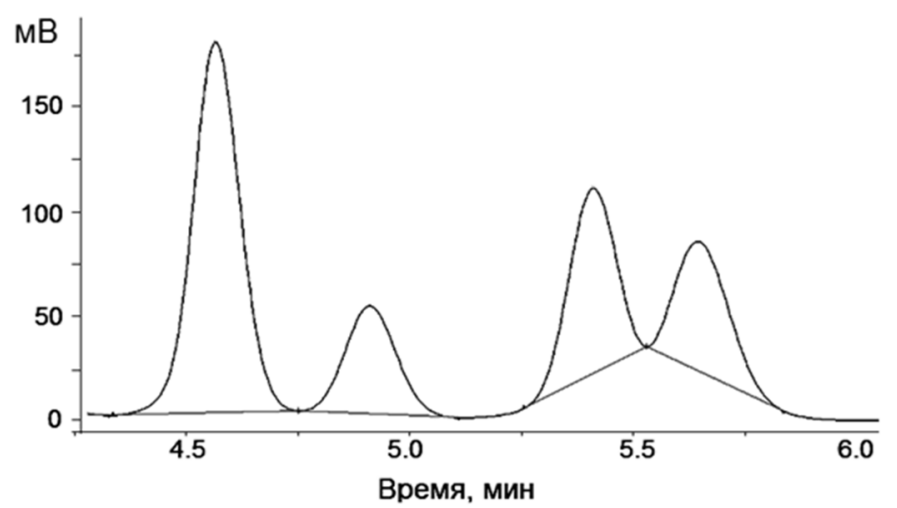

Рис. 1. Автоматическая разметка хроматограммы на одном из участков с неразделенными пиками в программе ChemStation Agilen

Цель настоящей работы - разработка способа дополнительной обработки хроматограмм с использованием программы Excel из пакета MS Office.

\section{Эксперимент}

При разделении триацилглицеринов льняного масла использовали хроматограф Shimadzu L20 с рефрактометрическим детектором; колонка 250×4.6 мм Kromasil 100-5С 18; подвижная фаза 20\% ацетонитрила в ацетоне, $0.8 \mathrm{~cm}^{3} / \mathrm{Mин.}$

Для разделения антоцианов плодов винограда использовали хроматограф Agilent 1200 Infinity с диодно-матричным детектором; колонка 150×4.6 мм Symmetry C18, 3.5 мкм; градиентное элюирование с использованием раствора А - 6 об. \% ацетонитрила и 10 об. \% муравьиной кислоты в воде и раствора Б - 20 об. \% ацетонитрила и 10 об. \% муравьиной кислоты в воде. Режим градиента: 0 мин - 0 \% Б; 20 мин 100\%Б, 30 мин. $100 \%$ Б, 31 мин - $0 \%$ Б, 40 мин - $0 \%$ Б; $0.8 \mathrm{~cm}^{3} /$ мин, температура термостата колонок $40^{\circ} \mathrm{C}$.

Экстракцию антоцианов из кожуры плодов винограда сорта Молдова готовили настаиванием в $0.1 \mathrm{M}$ водном растворе $\mathrm{HCl}$. Перед хроматографированием экстракт, отделенный от остатка фильтрованием через бумажный фильтр, очищали методом твердофазной экстракции на концентрирующих патронах Диапак С18 (БиохимМак СТ, Москва) [3]. В работе использовали льняное масло (ООО Ботаника). При обработке пиков использовали ПО: ChemStation 32 Agilent, LC и Мультихром 1.5 и MS Excel 2010.

\section{Обсуждение результатов}

Подготовим рабочий лист в программе MS Excel. Первые два столбца экспортируются из программы записи и обработки хроматограммы: 1-ый столбец - время, 2-ой столбец - отклик детектора. В верхней части вводим 5 свободных строк. Третий 
столбец - отклик детектора, измененный на указанную перед началом столбца величину (в ячейке C5) - для корректировки нулевой линии хроматограммы на выбранном участке $(=\mathrm{B} 6+\$ \mathrm{C} \$ 5)$.

Для компьютерного разделения пиков с $\mathrm{R}_{\mathrm{s}}$ менее 1 необходимо, прежде всего, задать форму пика. Гауссова функция (ГФ), представляющая форму идеального хроматографического пика в соответствие с методом теоретических тарелок $[4,5]$ имеет вид:

$$
y(t)=\frac{1}{\sigma \sqrt{2 \pi}} e^{-\frac{\left(t_{R}-t\right)^{2}}{2 \sigma^{2}}}
$$

где $t$ - время с начала записи хроматограммы, $t_{R}$ - время удерживания вещества, $\sigma-$ среднеквадратическое отклонение.

В соответствие с уравнением (1) в 2-3 столбцах (в зависимости от числа пиков, неразделенных на рассматриваемом участке хроматограммы) с некоторым отступом (например, под литерами Н, I, J для моделирования ГФ группы из трех пиков) указываем:

- в первой строке - номер пика;

- во второй строке - время удерживания (из хроматограммы);

- в третьей строке - численное значение знаменателя в уравнении (1);

- в четвертой строке - высоту пика («стартовое» значение которой также извлекается из хроматограммы).

В столбцах под литерами $\mathrm{H}$, I и J рассчитываем параметры каждой из хроматограмм, например, для ячейки K6=\$K\$4*(EXP(-((\$K\$2-A6)^2)/\$K\$3)), транслируя формулу на весь столбец. Данные столбцов H-J переносим в столбцы D-F, и рассчитываем сумму моделированных пиков D-F, стоблец G. Строим рисунок, на котором отмечены пунктирными линиями пики трех составляющих в виде гауссовых кривых, их сумму (красная пунктирная линия) и часть исходной хроматограммы с поправкой нулевой линии, с ограничением по началу и окончанию хроматограммы (в «Формате оси»). Затем подбираем высоту и ширину пиков для лучшего совпадения исходной хроматограммы и суммы моделированных пиков. Для контроля совпадения можно в отдельном столбце подсчитать сумму квадратов разностей двух последних хроматограмм, а еще лучше - контролировать степень совпадения двух кривых по еще одной разностной кривой. Полученный результат представлен на рис. 2. Расчет долей площадей пиков по данному методу привел к следующим результатам: пик №1 ( $\alpha$-линоленоат-дилинолеат) - 27.8\%; пик №2 (ди- $\alpha$-линоленоат-олеат) - 55.4\%; и пик №3 (ди$\alpha$-линоленоат-пальмитат) $-17.8 \%$ от суммы трех пиков. Полученные результаты мало отличаются от долей, рассчитанных при ручной обработке хроматограммы и разделением пиков вертикальной чертой (встроенная в ChemStation Agilent функция): 28.3, 53.7 и $18.0 \%$, соответственно. Но в предложенном варианте с использованием MS Excel можно рассчитать как число теоретических тарелок для каждого из пиков, так и разрешение пиков, $\mathrm{R}_{\mathrm{s}}$.

Из полученных результатов можно обратить внимание на то, что у пиков 1, 2 и 3 на рис. 2 «полуширина» изменяется не монотонно, - с максимумом для пика №2 (ди$\alpha$-линоленоат-олеата). Объяснение такого явления может быть следующим: для всех из указанных пиков возможно существование двух позиционных изомеров: ААБ и АБА. При этом вероятность биосинтеза каждого из них не одинакова, а их разделение в условиях обращенно-фазовой ВЭЖХ принципиально возможно [6]. Т.е. каждый из указанных пиков может быть суммой двух пиков с различающимися временами удерживания. При компьютерном моделировании нужно учитывать, что существует много причин для искажения формы хроматографического пика [7-11]. 


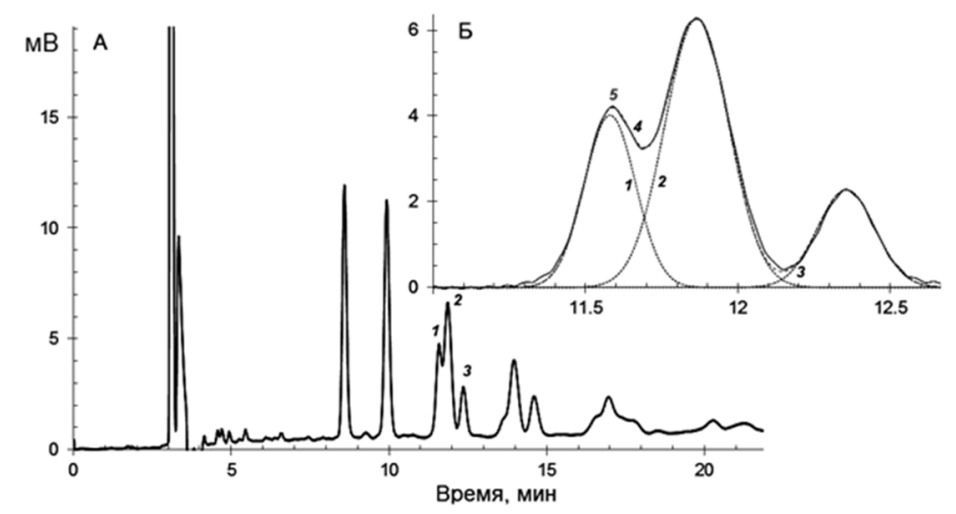

Рис. 2. Разделение триацилглицеринов льняного масла (А) и компьютерное разделение трех компонентов гауссовыми кривыми (Б)

Условия: колонка 250×4.6 мм, Kromasil 100-5C18, подвижная фаза, 20 об.\% ацетонитрила в ацетоне, $0.8 \mathrm{~cm}^{3} / \mathrm{Mин.}$

Моделирование формы пика в Excel представляет интерес, поскольку можно оценить степень отклонения формы пика, например, от гауссова распределения, или задать форму с учетом любого из известных вариантов искажения. Метод компьютерного моделирования позволяет предсказать изменение формы пика при соответствующем суммировании идеализированных пиков двух изомеров. Так, при $\mathrm{R}_{\mathrm{s}}=0.26$ с одинаковой площадью обоих изомеров (т.е. с одинаковой вероятностью биосинтеза) суммарный пик по-прежнему хорошо описывается гауссовой кривой, рис. 3, но с кажущейся эффективностью, сниженной в полтора раза по сравнению и исходной (для каждого из пика). Если соотношение между вероятностями биосинтеза изменяется до $2 / 3: 1 / 3$, то при том же $R_{s}$ отклонение суммы пиков от гауссова распределения становится очевидным - так, как если бы к пику добавили еще один небольшой пик с большим удерживанием, рис. 3Б. Это могло бы объяснить тонкие эффекты, полученные при обработке пиков триацилглицеринов, описанные ранее.

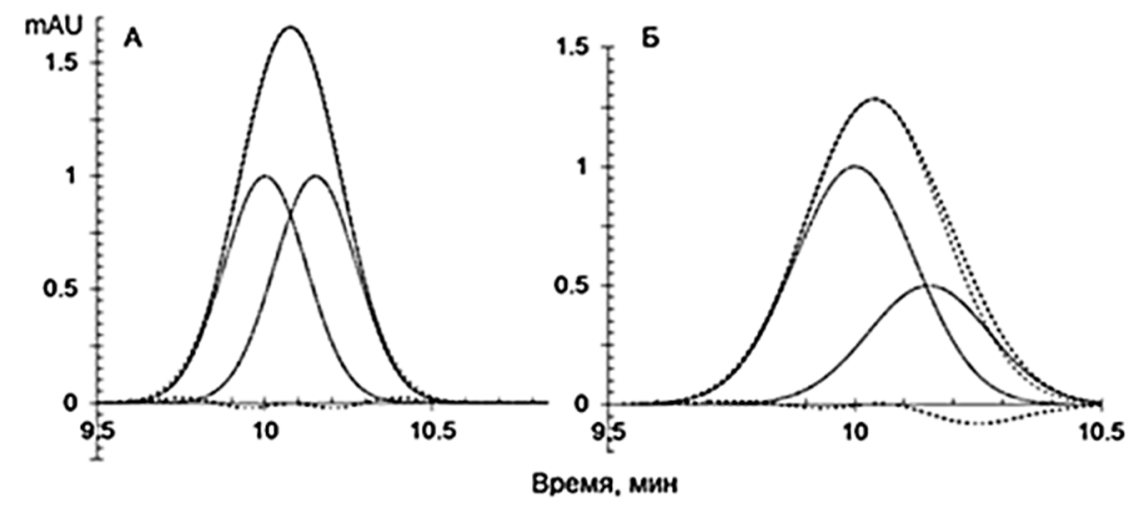

Рис. 3. Моделирование хроматограмм двух пиков с Rs $=0.26$ при количественном соотношении А $-0.5: 0.5$ и Б $-0.67: 0.33$.

Но отклонение от линейности в изотерме адсорбции (по крайней мере, на начальной стадии разделения) также может быть причиной искажения формы пика. Так, аналогичный анализ пиков три- $\alpha$-линоленоата, для которого нет изомеров и для ди- $\alpha$-линоленоата-линолеата (имеет два изомера) показал, что в каждом из случаев при моделировании пиков при помощи ГФ к результату желательно добавить еще один меньший пик с немногим большим удерживанием - это новое представление 
несимметричных пиков, рис. 4. Для пиков, искаженных выходом за пределы линейности изотермы адсорбции, степень и характер искажения зависят от стартовой концентрации вещества в пробе, поэтому единой формулы для их моделирования не существует.

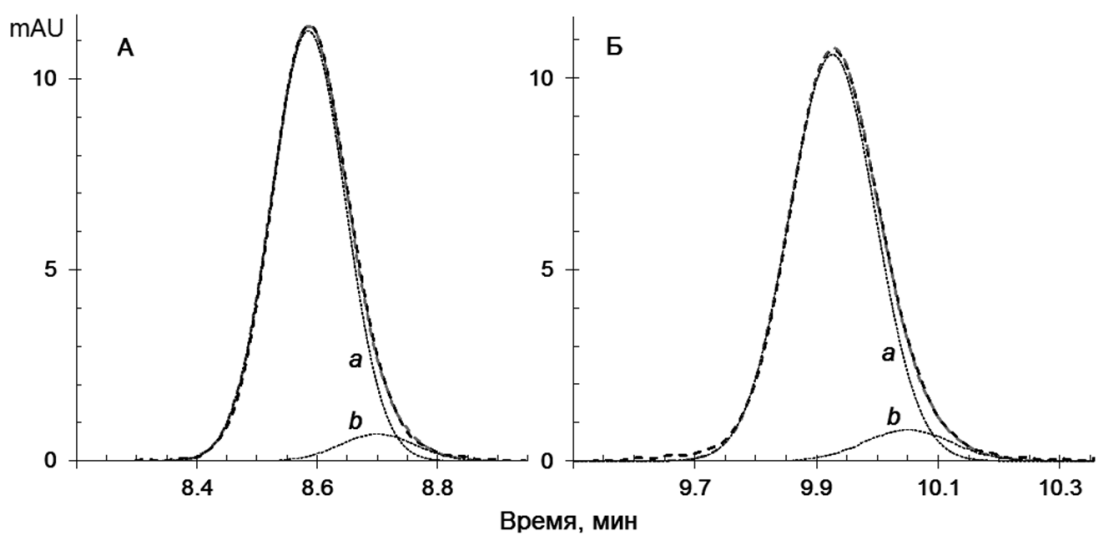

Рис. 4. Аппроксимация пиков триацилглицеринов из льняного масла двумя гауссовыми функциями gики: А - три- $\alpha$-линоленоата; Б - ди- $\alpha$-линолеата; $a$ - основная гауссова функция, $b-$ корректирующая гауссова функция.

Эффективность разделения пиков вертикальной чертой оказалась достаточно высокой при разделении пиков с близкими «полуширинами». Но необходимо дополнительно оценить эффективность такой процедуры для разделения с различной степенью уширения соседних пиков (т.е. с различным числом теоретических тарелок). Такие пики встречаются при ВЭЖХ антоцианов экстрактов гибридных сортов виноградов, в которых эффективность пиков 3-глюкозидов существенно выше эффективности пиков 3,5-диглюкозидов, рис. 5. В этом случае расчет показывает небольшое различие при оценке площадей по ГФ и прямого разделения вертикальной чертой, если более узкий пик предшествует более широкому: доля площадей пиков цианидин3 -глюкозида (1) и пеонидин-3,5-диглюкозида (2) составляет 6.4 и 10.3\% по первому варианту и 6.6 и 9.9\% по второму. При обратном порядке элюирования погрешности становятся большими: доля площадей мальвидин-3,5-диглюкозида (3) и петунидин-3глюкозида (4) составляет 57.9 и $25.4 \%$ по первому варианту и 49.9 и $33.6 \%$ - по второму.

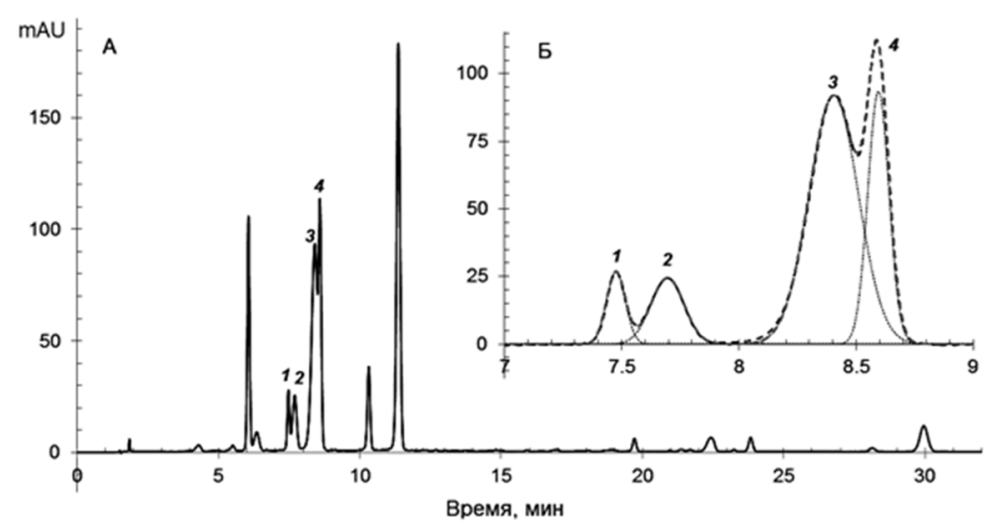

Рис. 5. Разделение антоцианов плодов винограда сорта Молдова

в градиентных условиях и представление не полностью разделенных соединений гауссовыми кривыми. А - полная хроматограмма; Б - фрагмент хроматограммы с не полностью разделенными цианидин-3-глюкозидом (1), пеонидин-3,5-диглюкозидом (2), мальвидин-3,5-диглюкозидом (3) и петунидин-3-глюкозидом (4). 
Рабочий лист позволяет легко определить число теоретических тарелок для каждого из пиков, форма которого моделирована ГФ. «Полуширина» пика, $\omega$, связана с дисперсией $(\sigma)$ уравнением:

$$
\omega=2 \sqrt{2 \ln 2} \cdot \sigma .
$$

Поэтому по величине знаменателя экспоненты, расположенного в третьей строке среди параметров пика, и времени удерживания - во второй строке, при расчете числа теоретических тарелок по формуле:

$$
N=5.54 \cdot\left(\frac{t_{R}}{\omega}\right)^{2}=8 \cdot \ln 2 \cdot \frac{t_{R}^{2}}{8 \cdot \ln 2 \cdot \sigma^{2}}=\frac{t_{R}^{2}}{\sigma^{2}}
$$

эффективность для каждого из моделированных пиков (т.е. для параметров выбранного столбца, например, L) рассчитывается по формуле: $N=2 \cdot \mathrm{L} 2^{\wedge}(2) / \mathrm{L} 3$.

\section{Заключение}

Таким образом, не полностью разделенные пики триацилглицеринов масел (на примере льняного масла), записанные при хроматографировании проб с относительно

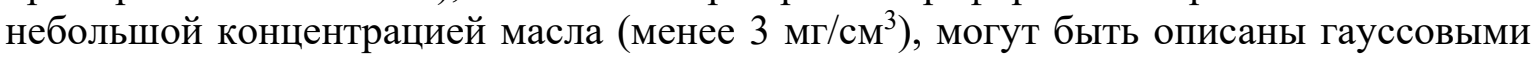
кривыми. При этом возможен расчет таких параметров как число теоретических тарелок, разрешение пиков, хотя результат количественного определения триацилглицеринов мало отличается от результата, полученного разделением близко элюирующихся пиков вертикальной чертой. Однако если ширины неразделенных пиков существенно различаются, то корректное соотношение между их содержанием может быть получено только после их разделения гауссовыми функциями. Установлено, что дополнительное уширение возможно в том случае, если пик объединяет два изомера (например, изомеров положения триацилглицеринов) положения с небольшим различием удерживания между ними. Эмпирически установлено, что небольшое отклонение пиков от гауссового распределения может быть компенсировано добавлением небольшого пика с большим вре3менем удерживания, параметры которого могут быть подобраны при компьютерном моделировании пика в программе MS Excel.

\section{Список литературы}

1. El-Hamdy A.H., Perkins E.G. // J. Amer. Oil Chem. Soc. 1981. Vol. 58. pp. 867-872.

2. Нгуен А.В., Дейнека В.И., Дейнека Л.А. // Вестник ВГУ. Сер. Химия. Биология. Фармация. 2019. № 1. С. 20-26.

3. Дейнека В.И., Кульченко Я.Ю., Чулков А.Н. и др. // Журн. физ. химии. 2019. Т. 93. С. 790-792.

4. Martin A.J.P., Synge R.L.M. // Biochem. J. 1941. Vol. 35. pp. 1358-1368.

5. Дейнека В.И. // Ж. физ. химии. 2004. Т. 78. C. 144-147.
6. Arnaud W.G. Sompila T., Héron S. et al // J. Chromat. B. 2017. Vol. 1041-1042. pp. 151-157.

7. Conder J.R. // J. HRC \& CC. 1982. Vol. 5. pp. 341-348.

8. Conder J.R. // J. HRC \& CC. 1982. Vol. 5. pp. 397-403.

9. Castellsa C.B., Castellsa R.C. // J. Chromat. A. 1998. Vol. 805. pp. 55-61.

10. Дейнека В.И., Сидоров А.Н., Дейнека Л.А. и др. // Сорбчионные и хроматографические прочессы. 2016. Т. 16. № 3. С. 384-389.

11. Дейнека В.И. // Ж. физ. химии. 2008. Т. 82. №1. С. 120-124. 


\title{
Utilization of MS Excel for processing of unresolved peaks on the chromatograms
}

\author{
(C) 2020 Deineka V.I. ${ }^{1}$, Turtygin A.V. ${ }^{2}$, Nguyen Van $\mathrm{Anh}^{3}$, \\ Oleinits E.Yu. ${ }^{1}$, Deineka L.A. ${ }^{1}$ \\ ${ }^{1}$ Belgorod National Research University, Belgorod \\ ${ }^{2}$ The National Institute of Accreditation, Moscow \\ ${ }^{3}$ Hanoi Pedagogical University 2, Hanoi, Vietnam
}

\begin{abstract}
Conventional software for the processing of chromatograms does not provide the possibility of peak simulation with a Gaussian function. This causes a problem for the correct calculation of the areas of unresolved peaks. The purpose of this paper was to propose a method for computer-generated simulation of the peaks with Gaussian functions in MS Excel. The parameters of the chromatogram (time and values of the detector responses) are imported into MS Excel. The peaks are simulated using Gaussian functions with manually selected parameters, including retention times (close to the experimental value), standard deviations, and peak heights to fit the section of the chromatogram with unresolved peaks. The processing ensures more exact calculation of the quantitative ratio of the substances, both for the peaks of similar width and for the peaks with markedly different effectiveness in the numbers of theoretical plates. Meanwhile, software with manual peak resolution by vertical divider (a built-in function of the software) is also suitable in the former case, although in the latter case it may provide incorrect results. The simulation was performed for the chromatograms of flax seed oil triacylglycerides and the anthocyanins of the fruit of the grape cultivar Moldova. The latter contains 3-glucosides and 3,5-diglucosides of common anthocyanins at the same time, which are markedly different in peak width at half height on the chromatogram. It was shown that some closely eluted pairs of compounds (such as positional triacylglycerols, for instance) can still be described by a Gaussian distribution, but the deviations increase in case of unequal quantities of the isomers. In this case, the resulting peak shape can be simulated using the main Gaussian with addition of another small one with a slightly greater retention. This type of simulation was found suitable not only for peaks with possible existence of positional isomers but also for a single-compound peak (e.g. tri- $\alpha$-linolenoate).
\end{abstract} analysis.

Keywords: HPLC, computer simulation of peaks, MS Excel, Gaussian, undivided peaks, quantitative

\section{References}

1. El-Hamdy A.H., Perkins E.G., J. Amer. Oil Chem. Soc., 1981, Vol. 58, pp. 867-872. https://doi.org/10.1007/BF02672960.

2. Nguyen Van Anh, Deineka V.I., Deineka L.A., Vestnik VGU. Ser. Himiya. Biologiya. Farmaciya, 2019, № 1, pp. 20-26.

3. Deineka V.I., Kul'chenko Ya.Yu., Chulkov A.N. et al., Russ. J. Phys. Chem. A, 2019, Vol. 93, No. 5, pp. 997-999 DOI: 10.1134/S003602441905008X.

4. Martin A.J.P., Synge R.L.M., Biochem. J., 1941, Vol. 35, pp. 1358-1368. doi: 10.1042/bj0351358

5. Deineka V.I., Russ. J. Phys. Chem. A, 2019, Vol. 78(1), pp. 91-93.

6. Arnaud W.G. Sompila T., Héron S. et al., $J$. Chromat. B, 2017, Vol. 104-1042, pp. 151-157. http://dx.doi.org/10.1016/j.jchromb.2016.12.030
7. Conder J.R., J. HRC \& CC., 1982, Vol. 5, pp. 341-348.

https://doi.org/10.1002/jhrc. 1240050702 .

8. Conder J.R. J. HRC \& CC., 1982, Vol. 5, pp. 397-403.

https://doi.org/10.1002/jhrc. 1240050802

9. Castellsa C.B., Castellsa R.C., J. Chromat. A, 1998, Vol. 805, pp. 55-61. https://doi.org/10.1016/S0021-9673(98)00042-9

10. Deineka V.I., Sidorov A.N., Deineka L.A. et al., Sorbtsionnye $i$ khromatograficheskie protsessy, 2018, Vol. 16, No 3, pp. 384-389. [in Russian].

11. Deineka V.I., Russ. J. Phys. Chem., 2008, Vol. $\quad 82, \quad$ pp. 108-111. DOI: $10.1134 / \mathrm{S} 0036024408010160$ 
Дейнека Виктор Иванович - профессор кафедры общей химии, д.х.н., Белгородский государственный национальный исследовательский университет, Белгород

Тутыгин Александр Владимирович - младший научный сотрудник Федеральное автономное учреждение «Национальный Институт Аккредитации», Москва

Нгуен Ван Ань - аспирант кафедры общей химии Белгородский государственный национальный исследовательский университет, Белгород

Олейниц Елена Юрьевна - аспирант кафедры общей химии Белгородский государственный национальный исследовательский университет, Белгород

Дейнека Людмила Александровна - доцент кафедры общей химии, к.х.н., Белгородский государственный национальный исследовательский университет, Белгород
Deineka Viktor I. - Professor of the Department of General Chemistry Belgorod state national research University, Belgorod, e-mail deineka@,bsu.edu.ru

Turtygin Alexander V. - Junior researcher Federal Autonomous institution "National Institute of Accreditation", Moscow, e-mail issanea@mail.ru

Nguyen Van Anh - post-graduate of the Department of General Chemistry Belgorod state national research University, Belgorod, e-mail Vananhkhoanosh2gmail.com

Oleinits Elen Yu. - post-graduate of the Department of General Chemistry Belgorod state national research University, Belgorod, e-mail 812887@,bsu.edu.ru

Deineka Lyudmila, Associate Professor of the Department of General Chemistry Belgorod state national research University, Belgorod 